\title{
Statins and Erectile Dysfunction
}

\author{
John B. Kostis ${ }^{(\mathbb{D}}$, Jeanne M. Dobrzynski(D) \\ Cardiovascular Institute, Rutgers Robert Wood Johnson Medical School, New Brunswick, NJ, USA
}

This is an Open Access article distributed under the terms of the Creative Commons Attribution Non-Commercial License (http://creativecommons.org/licenses/by-nc/4.0) which permits unrestricted non-commercial use, distribution, and reproduction in any medium, provided the original work is properly cited.

Sexual activity is an important part of quality of life of men and erectile dysfunction affects other dimensions of the individual including depression, sexual performance, and self worth. At age 40, approximately $40 \%$ of men are affected by erectile dysfunction. The rate increases to nearly $70 \%$ in men aged 70 years [1]. This problem has become magnified because of the current aging of the population after the control of communicable diseases. In addition to age, erectile dysfunction is associated with cardiovascular disease since the two conditions share similar risk factors, such as diabetes, metabolic syndrome, sedentary lifestyle, smoking, injuries, or surgery to the pelvic area or spinal cord, obesity, hypertension, hyperlipidemia, and depression [2]. Thus, the treatment of erectile dysfunction depends on the underlying etiology. Attention and amelioration to the extent possible of the conditions mentioned above is the first therapeutic approach. In the large majority of older persons with erectile dysfunction, phosphodiesterase isoenzyme 5 inhibitors are indicated and have been proven to be effective, welltolerated and may have additional benefits in idiopathic pulmonary hypertension, heart failure, and coronary heart disease. A common instrument for the evaluation of sexual function in men is the International Index of Erectile Function (IIEF), a validated self-administered questionnaire that has been psychometrically sound and linguistically validated in ten languages. This instrument ranks on a scale of one to five, five dimensions of sexual function (erection, orgasm, desire, intercourse satisfaction, and overall satisfaction) [3].

The coexistence of erectile dysfunction with cardiovascular disease and especially coronary heart disease stemming from the commonality of risk factors mentioned above has resulted in increasing use of statins in patients with erectile dysfunction. Also, patients without erectile dysfunction who qualify for statin therapy need to be reassured regarding the relationship of statin therapy with erectile dysfunction. Lifestyle modification is important for both cardiovascular health and improvement of erectile function. In some instances, it may be sufficient to reverse pre-existing abnormalities in function as well as structure. In all cases, lifestyle change is a necessary adjunct to pharmacologic therapy. Reducing body weight with caloric restriction and regular exercise, as well as avoidance of smoking, are important in reducing the risk of coronary heart disease and erectile dysfunction. Randomized studies of pharmacologic agents to reduce cholesterol were performed on background non-pharmacologic therapy and specific pharmacologic therapy for co-morbidities such as hypertension diabetes, etc. discussed above. The effect of lifestyle changes on erectile dysfunction was examined in a randomized control trial of 110 obese men aged 35 to 55 years with body mass index $\geq 30 \mathrm{~kg} / \mathrm{m}^{2}$. In this study, non-pharmacologic 
therapy resulted in improvement of IIEF from $13.9 \pm 4$ to $17.0 \pm 5, p<0.001[4]$.

In deciding on the appropriate statin therapy, after lifestyle modifications, it is important to classify the patients into two categories, those where primary prevention is needed vs. those with clinical evidence of cardiovascular disease where secondary prevention is needed. The current recommendation is to use high intensity statins for patients with clinical atherosclerotic disease and to make sure that the patients have access to medication and adherence is monitored. For patients with clinical evidence of cardiovascular disease (secondary prevention), high intensity statin treatment is recommended for most patients by the 2013 American College of Cardiology/American Heart Association (ACC/AHA) guidelines [5]. For primary prevention, statin therapy is recommended for those with lowdensity lipoprotein cholesterol (LDL-C) >190 mg/dL as well as for patients with diabetes. For patients with LDL-C between 70 to $189 \mathrm{mg} / \mathrm{dL}$ moderate or high intensity statin therapy is recommended if the 10-year atherosclerotic risk is $\geq 7.5 \%$, and moderate intensity statin therapy for risk between $5.0 \%$ to $7.4 \%$ risk. If the risk is lower than 5\%, treatment is individualized [5]. In our opinion, an LDL-C below $100 \mathrm{mg} / \mathrm{dL}$ is appropri- ate for primary prevention, and an LDL-C $<70 \mathrm{mg} / \mathrm{dL}$ for secondary prevention. In patients who are statinintolerant, proprotein convertase subtilisin/kexin type (PCSK9) antibodies may be used.

Pharmacologic therapy for erectile dysfunction includes phosphodiesterase-inhibitors in the great majority of cases. Phosphodiesterase-inhibitors exert favorable effects on the cardiovascular system. They have been approved in the USA for the treatment of pulmonary hypertension, and may have beneficial effects on angina, heart failure, and control of hypertension. Cardio-protection, vasodilation, reduction of inflammation, and decreased oxidative stress has also been reported. These agents cannot be used together with nitrates because of marked reduction in blood pressure [2].

Statins may improve erectile function since LDL$\mathrm{C}$ is associated with better endothelial function, and good endothelial function is important for an erection. In addition, statins may improve endothelial function through 'pleiotropic' effects including increased availability of nitric oxide, which is considered the principal mediator of penile erection, decreased oxidative stress, and anti-oxidant effects. On the other hand, statins may impair erectile function because they block the 3-hydroxy-3-methylglutaryl-coenzyme A (HMG CoA)

Statins and erectile dysfunction all randomized trials

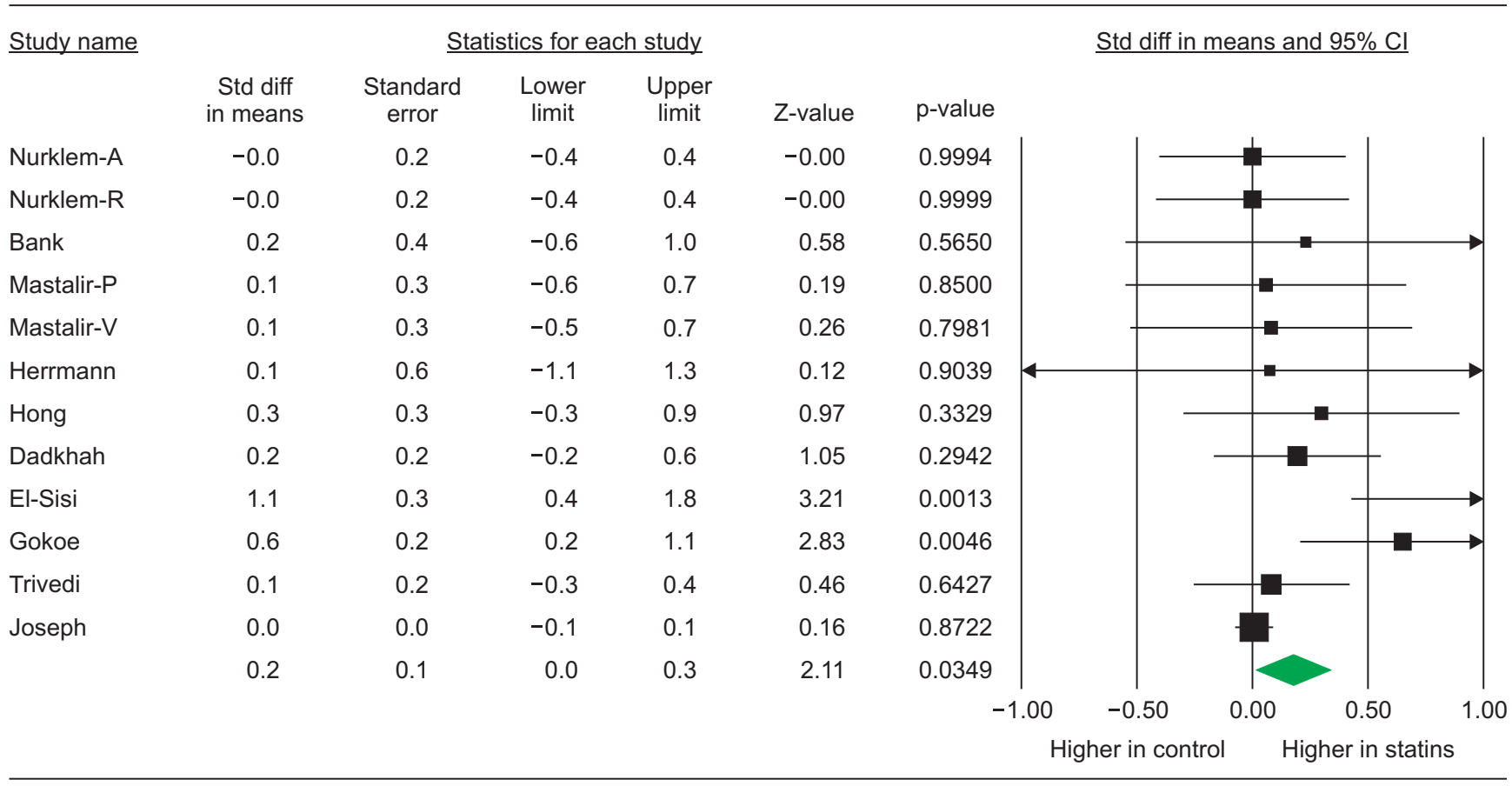

Fig. 1. Forest plot of randomized trials of the effect of statins on the International Index of Erectile Function Score. Solid squares have a size proportional to the inverse of the variance. Horizontal lines denote the $95 \%$ confidence interval (Cl) for individual trials. Std diff: standard difference. 
reductase at an early stage of cholesterol biosynthesis and thereby decrease the formation of testosterone, a hormone that is associated with increased sexual function [6]. We have performed a meta-analysis of randomized trials on the effect of statins on erectile dysfunction [6]. In a systematic search of the MEDLINE, the Web of Science, the Cochrane Database, and ClinicalTrials.gov, we identified 11 randomized trials to be included in the meta-analysis. This indicated a clinically relevant improvement in erectile function as measured by IIEF. Statins were associated with 3.4 units $(95 \%$ confidence interval [C], $1.7-5.0 ; p=0.0001)$ improvement compared to control. Limitations of this study, mentioned in our report, were the small number of subjects (713 patients) included the analysis, the short duration of follow-up, median 3 months, mean $4.2 \pm 3$ months, as well as possible publication bias, and incomplete adjustment for confounders. For these reasons, we are very interested in the recently published report from Heart Outcomes Prevention Evaluation-3 (HOPE-3) where Joseph et al [7] report on the effect of rosuvastatin on erectile function compared to placebo. This study included 2,153 patients, with 5.8 years of follow-up (three times as many patients and more than 15 times the average follow-up) as our previous metaanalysis. In HOPE-3, rosuvastatin was associated with small, improvement of IIEF compared to placebo. We updated the meta-analysis to include the data from HOPE-3 and the improvement of IIEF with statins remained statistically significant, but less pronounced (Fig. 1; standard difference in means 0.2; 95\% CI, 0.0-0.3; $\mathrm{p}=0.0349$ ). Random effects models were used in both the first and second (updated with HOPE-3) meta-analysis. Also, observational studies were excluded since they may be associated with residual confounding and undetected bias that may lead to unreliable associations.

In summary, data from currently available, randomized trials indicate a small improvement in erectile function with statins. This implies that further studies on erectile function with statins are needed.

\section{Disclosure}

The authors have no potential conflicts of interest to disclose.

\section{Author Contribution}

Research concept: Kostis JB. Statistical analysis: Kostis JB. Drafting of manuscript: Dobrzynski JM. Data extraction: Dobrzynski JM. Approval of final manuscript: all authors.

\section{REFERENCES}

1. Feldman HA, Goldstein I, Hatzichristou DG, Krane RJ, McKinlay JB. Impotence and its medical and psychosocial correlates: results of the Massachusetts Male Aging Study. J Urol 1994;151:54-61.

2. Ioakeimidis N, Kostis JB. Pharmacologic therapy for erectile dysfunction and its interaction with the cardiovascular system. J Cardiovasc Pharmacol Ther 2014;19:53-64.

3. Rosen RC, Riley A, Wagner G, Osterloh IH, Kirkpatrick J, Mishra A. The international index of erectile function (IIEF): a multidimensional scale for assessment of erectile dysfunction. Urology 1997;49:822-30.

4. Esposito K, Giugliano F, Di Palo C, Giugliano G, Marfella R, D'Andrea F, et al. Effect of lifestyle changes on erectile dysfunction in obese men: a randomized controlled trial. JAMA 2004;291:2978-84.

5. Stone NJ, Robinson JG, Lichtenstein AH, Bairey Merz CN, Blum CB, Eckel RH, et al. 2013 ACC/AHA guideline on the treatment of blood cholesterol to reduce atherosclerotic cardiovascular risk in adults: a report of the American College of Cardiology/American Heart Association Task Force on Practice Guidelines. Circulation 2014;129:S1-45.

6. Kostis B, Dobrzynski JM. The effect of statins on erectile dysfunction: a meta-analysis of randomized trials. J Sex Med 2014;11:1626-35.

7. Joseph P, Lonn E, Bosch J, Lopez P, Zhu J, Keltai M, et al. Long-term effects of statins, blood pressure-lowering, and both on erectile function in persons at intermediate risk for cardiovascular disease: a substudy of the Heart Outcomes Prevention Evaluation-3 (HOPE-3) randomized controlled trial. Can J Cardiol 2018;34:38-44. 